\title{
Application-Specific Discriminant Analysis of Cardiac Anomalies Using Shift-Invariant Wavelet Transform
}

\author{
Ritu Singh, Guru Gobind Singh Indraprastha University, India \\ Navin Rajpal, Guru Gobind Singh Indraprastha University, India \\ Rajesh Mehta, Thapar Institute of Engineering and Technology, India
}

\begin{abstract}
Automatic arrhythmia detection in electrocardiogram (ECG) using supervised learning has gained significant considerations in recent years. This paper projects the performance analysis of classifiers such as support vector machine (SVM), extreme learning machine (ELM), and k-nearest neighbor $(\mathrm{KNN})$ with efficient time utilization showing multi-classification for specific medical application. The wavelet double decomposition is used to show the shift-invariant use of dual-tree complex wavelet transform for noise filtering and beat segmentation is done to extract 130 informative samples. Further, the linear discriminant analysis is applied to dimensionally reduce and elite the 12 most relevant features for classifying normal and four abnormal beats collected from MIT/BIH ECG database. The proposed executed system distinguishes SVM, ELM, and KNN with percentage accuracy of $99.8,97$, and 99.8 having classifier testing time as $0.0081 \mathrm{~s}, 0.0031 \mathrm{~s}$, and $0.0234 \mathrm{~s}$, respectively. The simulated experimental outcomes in comparison with existing work yields adequate accuracy, and computational time.
\end{abstract}

\section{KEYWORDS}

Dual-Tree Complex Wavelet Transform, Electrocardiogram, Extreme Learning Machine, K-Nearest Neighbor, Linear Discriminant Analysis, Medical Applications, Support Vector Machine

\section{INTRODUCTION}

Biomedical signals represent the electrical dynamism of the human body. The medical experts study these signals to acquire vital information regarding the health challenges. Electrocardiogram (ECG) is a non-invasive electrical signal whose automated analysis has motivated the current medical diagnostic scenario for user applications to handle at ease. These applications deal with free ECG check-up camps, Intensive Care Unit (ICU), Emergencies, and mobile fast track devices. Despite having difficulties extracting accurate and reliable ECG information, the biological systems eager to shift towards automation and E-health programs. Many upcoming novel types of research are encouraged to resolve these issues, keeping in mind the appropriate ECG data acquisition, meticulous feature extraction, ace classifiers, computational cost, and dynamic memory management.

ECG pattern is a simple and useful tool for analyzing cardiac disturbances as it has set operations for a healthy heart. The depolarization-repolarization cycle of heart chambers shows the exact time evolution of the P-QRS-T pattern, as shown in Figure 1. R-peak is the most prominent feature which 
helps in recognizing the heartbeat count. Every ECG pattern is unique and different according to the human system (Mirvis and Goldberger, 2001). Any change in the morphological and temporal characteristics presents malfunctioning of heart, which leads to arrhythmia. An arrhythmia is an irregularity in the cardiac rhythm. It disturbs the workflow of other organs like the brain, lungs, etc. Foremost, the health check requires ECG testing and analysis. In this paper, the discriminant analysis of shift-invariant ECG features is done with aspired classifiers to make an automated proposal for specialized medical applications.

\subsection{Literature Review}

For the upcoming new technology era, the ECG diagnosis has to be efficient and computer-aided. Many research works have come up taking feature recognition and classification as a challenging task to fulfil these requirements. The automatic ECG analysis requires three phases: ECG signal enhancement, feature extraction with beat segmentation, and classification. The signal enhancement, i.e., noise filtering techniques and feature extraction, generally includes the signal decomposition, feature recognition, and signal reconstruction using required attributes. Many existing studies, like digital filters, Wavelet transform (WT), higher-order statistical concepts, and threshold approaches based on time, frequency, and time-frequency resolutions, have contributed to this area. The frequency and time observations go side by side. At lower margins, the signal data will be smooth in the frequency domain and blemished in the time domain and vice-versa. The octave bands are also slender, whereas, at a higher frequency, the octave bands are outstretched.

Multi-Rate digital processing of the ECG signal is reported using filter banks used for getting uniform frequency sub-bands (Afonso et al.,1999). The real-time multi-classification of ECG beats with R-peak detection, and other morphological features are depicted showing performance evaluation as well (Raj et al.,2015; Mitra et al., 2006). The time analysis has few shortcomings related to the fiducial onset and offsets points of ECG wave such as standard variability, uncertainty in finding locations, and boundaries. The changes in normal ECG and QRS complex is observed using Fourier Transformation (Minami et al.,1999).

This spectral analysis was not able to provide timely information related to frequency components. The WT based time-frequency analysis proves to be more potent than constant window short time FT (STFT) for non-stationary signals. In (Kaur et al. 2016), ECG was characterized using discrete WT and inferred that the results outperform Principal Component Analysis (PCA). According to (Inan et al. 2006), WT with timing features and neural networks for classification can discriminate normal and arrhythmic conditions of ECG. The wavelet works on the principle of using a broader window at the low and narrower window at high frequency. In all wavelet basis functions, the Daubechies proof to be the best for using non-linear ECG signal feature extraction (Singh et al., 2018). In (Martis et al. 2013), along with DWT extracted features, successfully applied PCA, LDA, and Independent Component Analysis (ICA) for dimension reduction and focused on only twelve prime significant features. In (Acharya et al. 2017) used HOS and cumulants of each ECG beat to get a feature dataset and used PCA for lower dimensions to become compatible for further classification. Having an excellent approach, DWT still lacks directional selectivity and invariant energy at the output. The implementation of DTCWT for noise removal and feature extraction (Thomas et al., 2015) covered this drawback. The feature extraction steps lead to the classifier to analyze the correctly classified and misclassified beats.

Many classifying approaches have shown their compatibility with feature extraction and reduction algorithms. These are Artificial Neural Networks (ANN), KNN, SVM, ELM, and other deep learning techniques. In (Shadmand et al., 2016), applied Block based NN(BPNN) using the PSO algorithm is involved, and they achieved an accuracy of 97\%. In (Plawaik, 2018), the use of spectral density measure of ECG fragments with an evolutionary neural network i.e., a genetic algorithm trained SVM outperforms other machine learning classifiers. The biomedical signals have recently shown good results with deep learning architecture. These show a single end to end structure consisting of 
Figure 1. Generation of P-QRS-T of Electrocardiogram

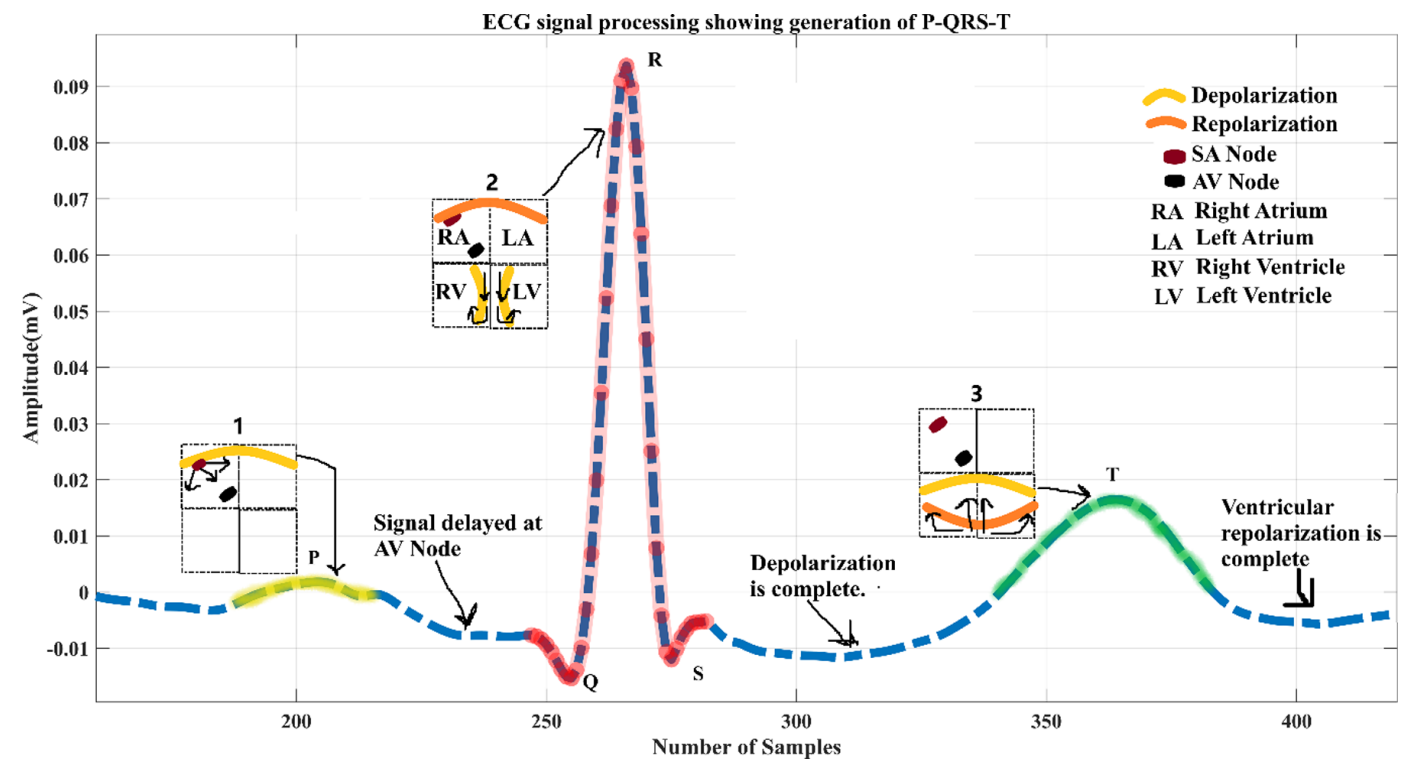

both feature extraction and classification. The convolutional neural network (CNN), a deep learning approach is demonstrated for noisy and noise-free ECG achieving an accuracy of $93.53 \%$ and $95.22 \%$ respectively for normal and myocardial infarction (MI) signal (Acharya et al., 2017). In (Oh et al., 2018) classified cardiac irregularities using a combined approach of a convolutional network and long short-term memory (LSTM).

\subsection{Research Focus and Motivation}

Nowadays, with the enhanced demand for automated Electrocardiography systems, a reliable, affordable, and non-invasive analysis of the user's ECG is required. These systems can be designed according to the requirements of applications such as computational cost, memory required, efficient time management, and medical experts' involvement. This flexibility is possible if the ECG feature extraction is very accurate and lossless as well as the classifier's choice for a particular use is judicially justified in processing time. The current research is motivated to focus on the following directions:

1. The use of wavelet double decomposition involving shift-invariant DTCWT to have no information loss during ECG signal processing, noise filtering, and ECG beat segmentation.

2. The implementation of the linear discriminant concept for inter and intra space analysis of five different ECGs.

3. The choice of classifiers according to the essential requirement of application for ECG check-up.

According to the suggestions given by the medical experts (mentioned in acknowledgment), the authors chose the classifiers. In the previous work, (Singh et al., 2019) have addressed ECG into normal and abnormal beats with successful feature extraction and classification using a hybrid approach and diverse classifiers. Also, they (Singh et al., 2020) have implemented a five-beat ECG classification using DWT, kernel PCA, and kernel ICA. 


\subsection{Paper Organization}

The remaining sections are set in sequence as in the material and methods section 2 , the material required, i.e., ECG arrhythmia database, is presented with procedural steps for ECG feature extraction using DTCWT, LDA, and with the brief of classifiers like KNN, SVM and ELM. The next is a result section 3 that presents the experimental outcomes of the proposed framework with simulations and performance calculations. And it is followed by application-specific suggestions for performance evaluation, and then results are deliberated in the discussion section 4. Finally, the last part, i.e. section 5 concludes with future aspects.

\section{MATERIAL AND METHODS}

The current application-based ECG analysis scheme is shown in Figure 2. The central concept is to develop an automated framework to classify ECG arrhythmias for health care services for everyday users and medical experts.

\subsection{ECG Database Used}

The most popular benchmarked, MIT/BIH ECG records, and beats are used for the performance analysis (Moody and Mark,2001). Table 1 mentions the ECG records and the required ECG beats for the research. The data partitioning involves 1400 training size and 600 testing size under crossvalidation scheme.

\subsection{Proposed ECG Classification Approach}

The systematic flow for regular and four types of irregular ECG beats analysis is shown in Fig. 2 and Fig. 3 displays the flowchart presenting the pre-processing and feature extraction of ECG beats as:

Step 1: Read ECG record (.dat format) from the MIT/BIH arrhythmia database.

Step 2: ECG Pre-processing:

- Normalize the amplitude of the ECG signal to compute zero mean and unit standard deviation.

- Low-frequency Noise (Base Line Drift) Filtration: Apply the multiresolution attribute of DTCWT by acquiring FIR filters for the following states. This procedure is done by decomposing up to level 9 using dB4, which results in nine details (D1 - D9) and one approximation coefficient (A9). In the A9 coefficient, the frequency range varies as $0-0.351 \mathrm{~Hz}$. By entirely discarding A9 and reconstructing the ECG signal using the remaining D1 to D9 coefficients, it makes baseline drift to zero position. It is hence removing irregularities from the signal.

- High-frequency Noise (Power Line Interference) Filtration: Again, apply DTCWT decomposition of level 4 using Db4. The high-frequency noise is contaminated in detail coefficients (D1 to D4). Denoise every detail coefficient according to the universal Donoho threshold method. Reconstruct enhanced ECG signal using inverse DTCWT coefficients, including modified D1 to D4 and approximation coefficient A4.

Step 3: ECG Feature extraction:

- Separate regular and irregular beats from ECG records using signal processing techniques. These beats are subjected to R- peak detection by finding maximum amplitude selection.

- Execute beat segmentation by collecting 130 data samples, including R-peak. This segment comprises of P-QRS-T wave of each ECG beat, making the feature dataset $(2000 \times 130)$.

Step 4: ECG Feature reduction: 
Table 1. Required Dataset Information

\begin{tabular}{|c|c|}
\hline Information & Required Data \\
\hline $\begin{array}{c}\text { Database } \\
\text { Number of Observations (Total) }\end{array}$ & $\begin{array}{c}\text { MIT/ BIH } \\
2000\end{array}$ \\
\hline Normal Beats (NB) & $400,(101--103--122--234)$ \\
\hline Abnormal ECG Beats (AB) & 1600 \\
\hline$\bullet$ Paced & $400,(102--107--104--217)$ \\
\hline$\bullet$ LBBB & $400,(109--111--214--207)$ \\
\hline$\bullet$ RBBB & $400,(118--124--231--212)$ \\
\hline PVC & $400,(119--208--200--233)$ \\
\hline Cross Validation & Hold-out $(0.30$ as a scalar parameter $)$ \\
\hline NumTestSets & 1 \\
\hline Train Size & 1400 \\
\hline Test Size & 600 \\
\hline
\end{tabular}

Apply LDA on this extracted ECG feature dataset to get dimensionally reduced features (2000 $\times 12)$.

Step 5: ECG Classification and its Application:

- Perform classification using KNN, ELM, and SVM using 1400 beats for training and 600 beats for testing of ECG feature dataset $(2000 \times 12)$.

- Calculate accuracy and classifier testing time. Choose medical applications according to the experimental outcomes and suggestions from experts.

The amplitude normalization is done using,

$$
\operatorname{norm}_{-} \mathrm{s}=\mathrm{s}-\min (\mathrm{s}(:))
$$

$\operatorname{norm}_{-} \mathrm{s}=$ norm $_{-} \mathrm{s} \cdot / \max (\mathrm{s}(:))$

Baseline drift gets incorporated in the ECG signal during the breathing of a person, and it is in a range of $0.15 \mathrm{~Hz}-0.3 \mathrm{~Hz}$. This interference can disturb the original ECG signal by changing zero origin. So, it needs elimination to keep morphological statistics unchanged. The power line interference disturbs ECG at a higher frequency of range $47 \mathrm{~Hz}-53 \mathrm{~Hz}$. It adds unwanted information in the signal.

In the proposed method, DTCWT double decomposition using Daubechies wavelet (Db4) is used to filter low-frequency and high-frequency noise, as shown in Fig. 3. The choice and level of wavelet are based on similarity and energy spectrum to match the original ECG signal.

For thresholding, the detail coefficients, the "Wave Shrink" known as the universal threshold is applied as, where $\sigma$ standard deviation, and M represents the size of the ECG signal at any detail decomposition or level as reported in (Georgieva-Tsaneva \& Tcheshmedjiev, 2013). The DTCWT denoising technique is also proved in (Prashar, Sood, \& Jain, 2018), and it shows the improvement in Signal to Noise ratio (SNR) from $-4.9666 \mathrm{~dB}$ to $27.7500 \mathrm{db}$. 


\subsection{Dual-Tree Complex Wavelet Transform (DTCWT)}

The use of wavelet and its operations has been a promising world for biomedical signals that continuously change its time, frequency, and morphological features (El B'charri et al., 2017). DTCWT has shown significant results in comparison with discrete WT as it uses the concept of Fourier Transform i.e., of complex-valued oscillating wavelet, whereas DWT uses the real-valued concept. The implementation of DTCWT as (Kingsbury N., 2001) shows:

- The amplitude does not oscillate and makes a smooth envelope.

- The magnitude is shift-invariant.

- The wavelet coefficients do not require anti-aliasing during reconstruction.

- Good directional selectivity and controlled redundancy factor for 1D signals.

DTCWT uses two functions, namely complex-valued wavelet $(\phi \mathrm{w})$ and wavelet scaling $(\phi s)$ functions. Furthermore, their real and imaginary components make Hilbert Transform pair having a 90॰ phase difference. The dual-tree structure is formed of two DWTs where one DWT tree forms the real part and another form imaginary part. The sampling rate is increased twice for every level, maintaining shift-invariant. The real part filters and imaginary part filters compose analytical filter design, and both execute parallelly. These filter banks consist of low and high pass techniques. For ECG records, DTCWT is used for denoising i.e., removing motion effects and other interferences. The denoising is implemented by signal decomposition and reconstruction.

The odd /even symmetric filters (Farras) are used for the first phase, and Q-shift filters (Kingsbury) are employed with the relationship of filters used is depicted as

$$
G_{e c g}^{B T r}(p)=g_{e c g}^{A T r}(p-0.5)
$$

where $g_{e c g}^{A T r}$ and $G_{e c g}^{B T r}$ both are low pass filters belonging to the upper and the lower filter banks, respectively. The transform uses Farras nearly symmetric filters for the first level and Kingsbury

Figure 2. Proposed Application based ECG Analysis framework

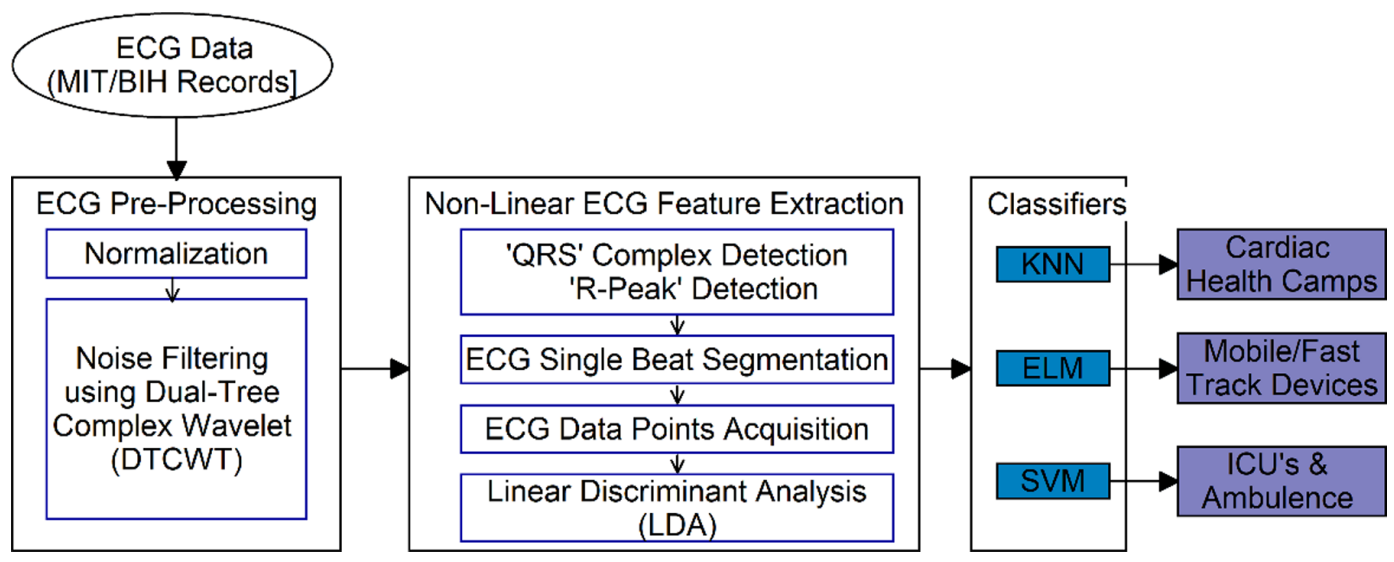


Q-shift filters with length 10 for subsequent levels. Table 2 presents the maximum noise drop by ECG signal while implementing DTCWT. The noise drop feature helps find the total noise content in the signal, which is proficiently excluded from it. Moreover, Figure 4 demonstrates the DTCWT implementation of ECG record number 101.

\subsection{Linear Discriminant Analysis (LDA)}

It is very decisive to cast relevant features necessary in training dataset for classification. The process is called dimensionality reduction. Fisher's LDA or normal discriminant analysis (NDA) is a dimension reduction method with a wide range of statistics and pattern recognition applications.

LDA approach helps training dataset by

- Avoid overfitting.

- Increase in simplicity.

- Increase computational efficiency.

LDA is considered as supervised data pre-processing method as it finds the feature subspace for optimizing group separability and reduce dimensions (Duin \& Loog, 2004). LDA's goal is to reduce the inner distance of the same class to a minimum and maximize the distance between multi classes. It uses a linear transformation to shift the original feature space to lower dimensions. It defines $\mathrm{Z}$ as a transformation matrix with a multi-class scatter matrix, and the same class scatter matrix. $T_{S}$ is the summation of covariance matrices which is equated as

$$
T_{S}=\sum_{p=1}^{N} \sum_{q=1}^{n i}\left(x_{q}^{(p)}-m_{p}\right)\left(x_{q}^{(p)}-m_{p}\right),
$$

where $x_{q}^{(p)} \in \Re^{z}$. Here $z$ represents the feature space dimension, $p$ is the class count and $q$ is the sample number of the respective class. Also $m_{p}$ mean of the mentioned class, $n_{i}$ is the number of samples in the class and $N$ is the total number of classes. For the current work, $N=5$ and $n_{i}=$ 400 .

$$
T_{M}=\sum_{p=1}^{N} n_{i}\left(m_{p}-m_{A l l}\right)\left(m_{p}-m_{A l l}\right)^{T}
$$

where $m_{A l l}$ is the mean of all five classes. $T_{M}$ is the summation of the squared distance calculated between the mean of each class and all five classes in this current study.

Finally, to achieve maximization, Fisher criterion can be expressed as

$$
J(Z)=\frac{Z^{T} T_{s} Z}{Z^{T} T_{M} Z}
$$

This is the ratio of new feature pace for the same class and for all five classes. To obtain the reduced dimension vector $y$, the decomposition of eigenvalues is applied and the equation used is 
Figure 3. Proposed pre-processing and feature extraction flow graph

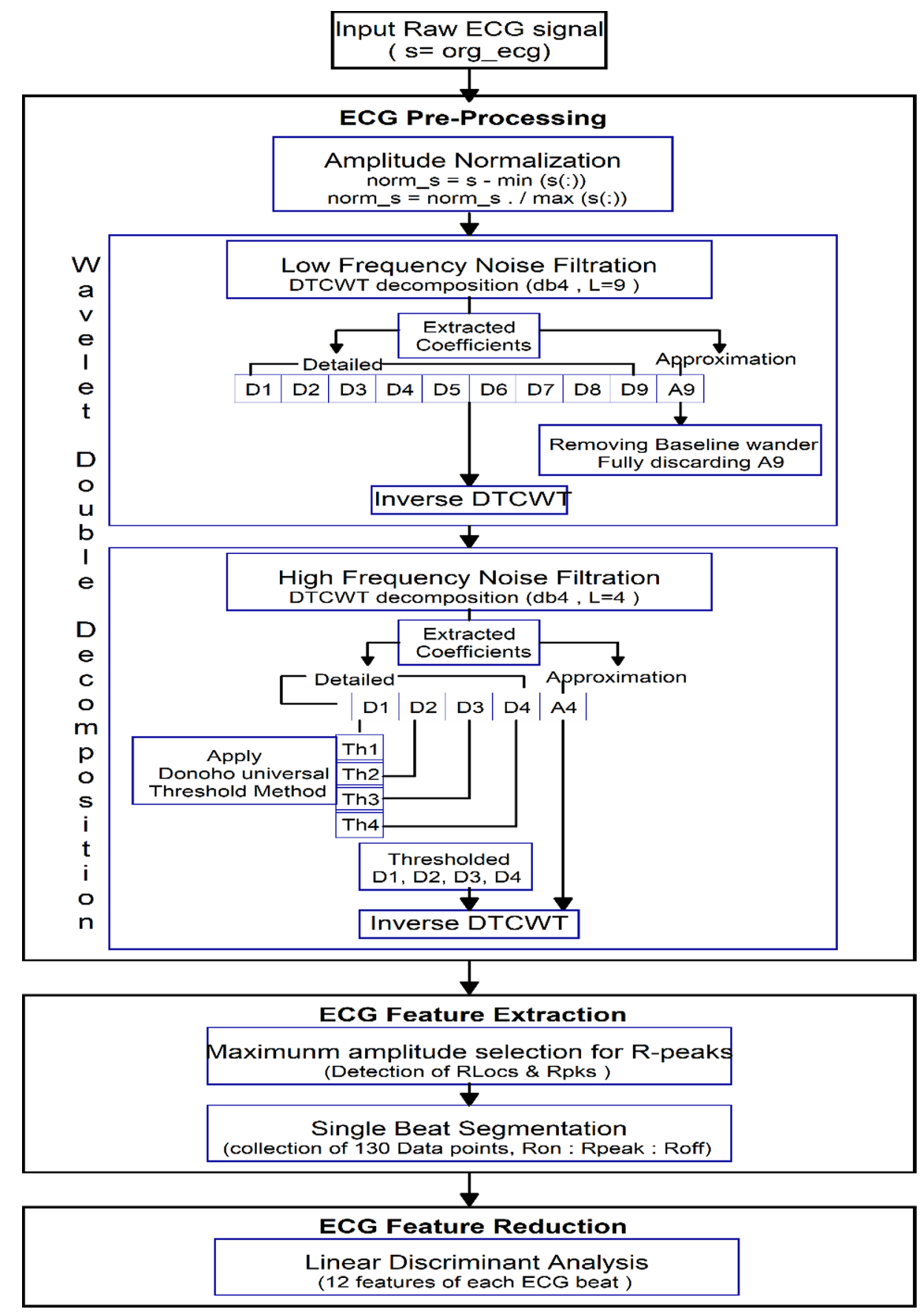

$y=Z^{T} x$

The advantages of using LDA on extracted ECG feature dataset are 
Table 2. Maximum noise drops from ECG signal using DTCWT filters

\begin{tabular}{|c|c|c|c|}
\hline ECG Record No. & Max. Noise Drop & $\begin{array}{c}\text { ECG Record } \\
\text { No. }\end{array}$ & Max. Noise Drop \\
\hline 101 & $1.0270 \mathrm{e}-15$ & 214 & $5.2180 \mathrm{e}-15$ \\
\hline 103 & $4.7740 \mathrm{e}-15$ & 207 & $4.2188 \mathrm{e}-15$ \\
\hline 122 & $1.7902 \mathrm{e}-15$ & 118 & $3.5527 \mathrm{e}-15$ \\
\hline 234 & $4.6629 \mathrm{e}-15$ & 124 & $4.9405 \mathrm{e}-15$ \\
\hline 102 & $4.0523 \mathrm{e}-15$ & 231 & $4.3299 \mathrm{e}-15$ \\
\hline 107 & $5.1070 \mathrm{e}-15$ & 212 & $4.3299 \mathrm{e}-15$ \\
\hline 104 & $4.4964 \mathrm{e}-15$ & 119 & $5.1070 \mathrm{e}-15$ \\
\hline 217 & $4.6074 \mathrm{e}-15$ & 208 & $5.2736 \mathrm{e}-15$ \\
\hline 109 & $4.2188 \mathrm{e}-15$ & 200 & $4.3299 \mathrm{e}-15$ \\
\hline 111 & $4.1633 \mathrm{e}-15$ & 233 & $4.8850 \mathrm{e}-15$ \\
\hline
\end{tabular}

- It easily handles the intra-space frequencies which are unequal

- It helps in performing on the randomly generated test dataset.

- It selects prime significant features of the ECG dataset. They help in formulating the ratio of inter-class variance to the intra class variance for maximum separability.

- It efficiently implements kernel tricks for non-linear transformation as well as classification.

In this paper, LDA is used to reduce the ECG feature dataset $(2000 \times 130)$ to the relevant feature dataset $(2000 \times 12)$. The six prime significant components are presented in Figure 7 .

\subsection{Classification Techniques}

\subsubsection{K-Nearest Neighbour (KNN)}

$\mathrm{KNN}$ is an instant based classifier that executes its operations according to the distance measure or some similarity function. The key benefits and the steps of using KNN as a supervised classifier in this current study are shown as:

- KNN classification is based on the training dataset. More training data, more probability of finding closest training points in the feature space. This concept demonstrates that an object from unknown testing data is classified according to its distances from the nearest cluster. (Venkatesan et al., 2018), has shown that KNN provides effective outcomes for ECG classification.

- KNN easily handles the dimensional reduction on feature dataset. Moreover, it has remarkable results with kernels.

- The classifier learning time is much less than other used classifiers, which lowers the computational cost. Moreover, KNN is easily deployed and adjusted in a resource-limited environment. KNN is the best-suited classifier for mass ECG check-ups where the number of training data is more.

The working condition of KNN depends on two factors one is $\mathrm{k}$, and the other is distance metric. For the current research work, $\mathrm{k}$ is taken as 3, and the ruler distance i.e., Euclidean distance metric is selected. For biomedical signals having feature extraction with reduced dimensions, KNN shows high accuracy, less learning time, and easy implementation. 


\subsubsection{Support Vector Machine (SVM)}

SVM as a classifier, shows remarkable performance for both two-class or multi-class analysis. SVM supports linear and non-linear classification (Gunn et al.,1998).

The working strategy of SVM is

- The first important step is to construct the optimal hyperplane of N Dimensions. It should distinguish a group of vectors of one category from lying another side of the hyperplane.

- Recognize support vectors (SVs) that are near hyperplane.

- The hyperplane should be oriented in such a way that the boundary or margin between SVs is maximized.

- In this maximal margin hyperplane, different kernel functions can be employed to execute nonlinear mapping. For classification procedure, SVM creates a non-linear decision limits in the input ECG feature space. This is also known as Structural Risk Minimization. Kernel function maps the dataset in different spaces.

The noise interference makes non-linear data that is not linearly separable. The kernel function used in the proposed work is the Radial basis Function, which maps the data into higher dimensional space for classification.

Figure 4. Original ECG record no. 101 with normalized, base line drift removed and DTCWT denoised signal
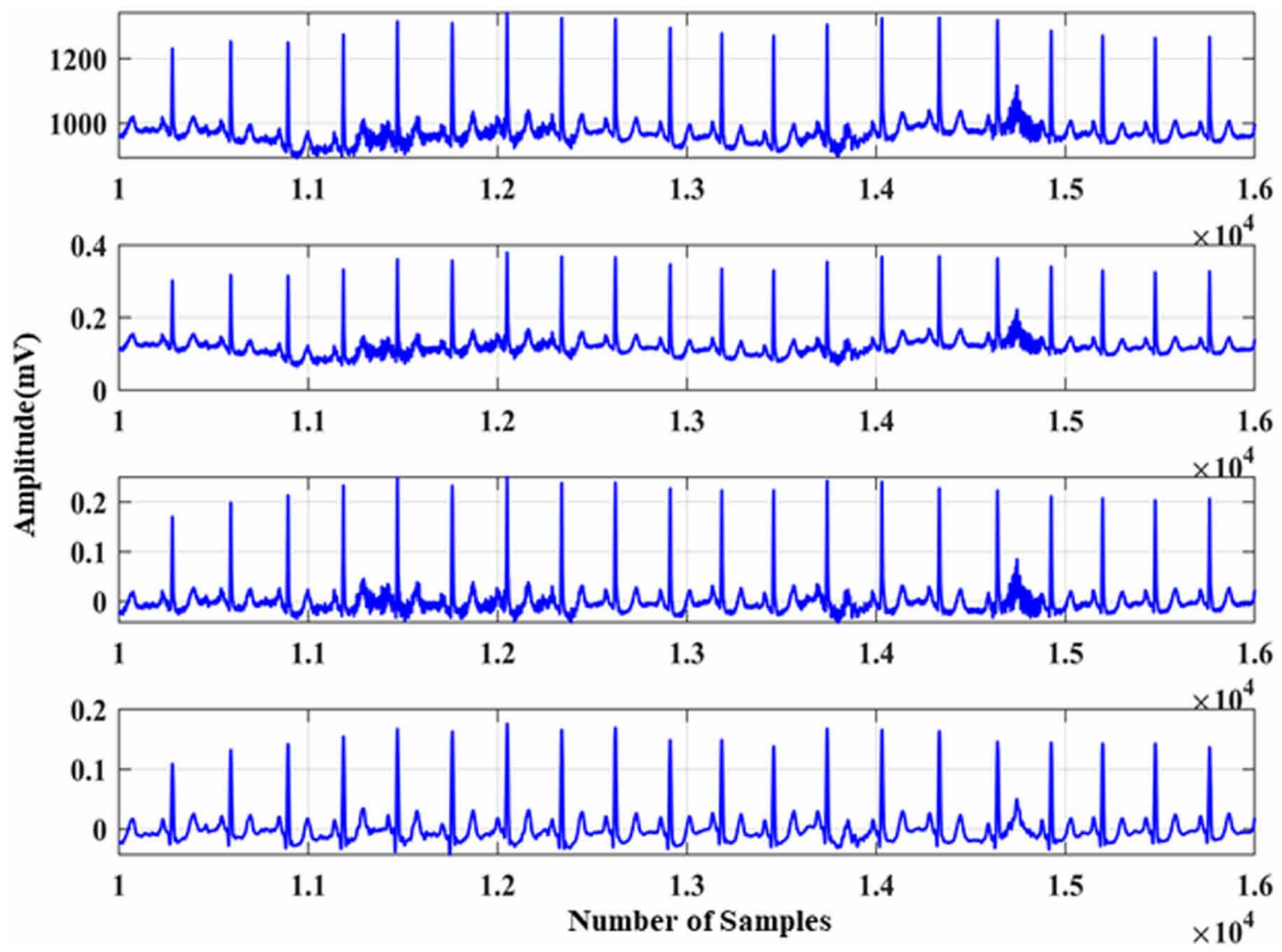
(a)

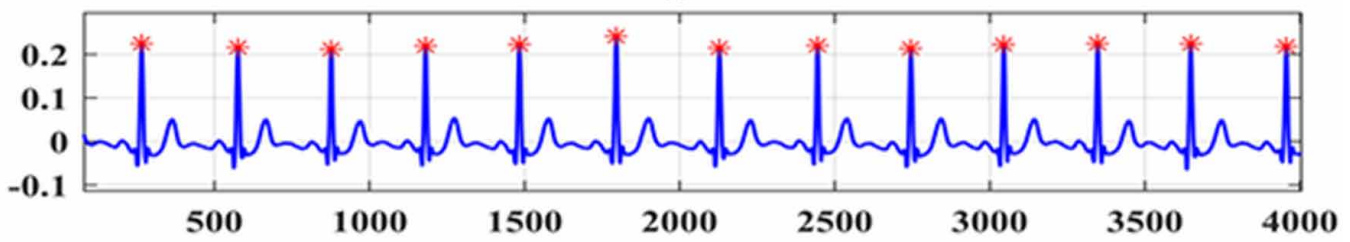

(b)

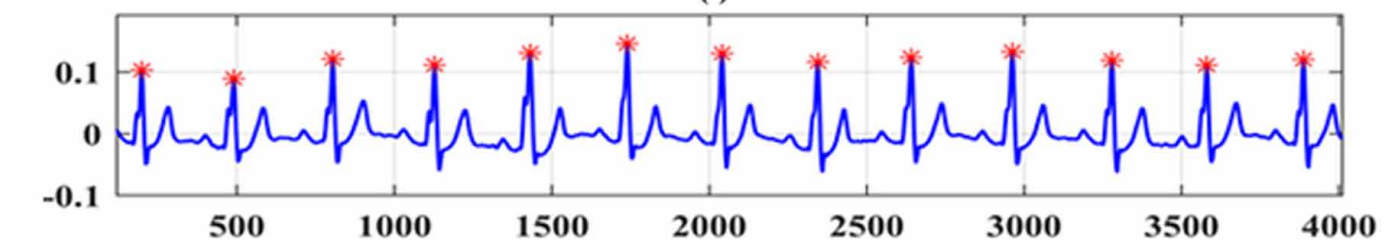

(c)

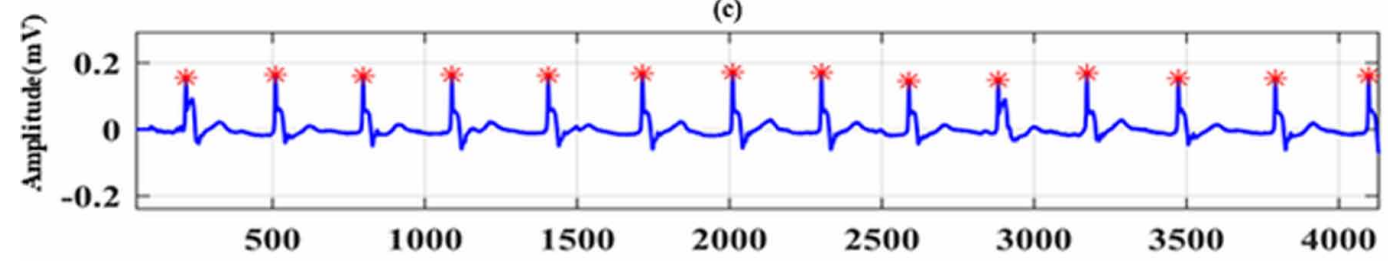

(d)

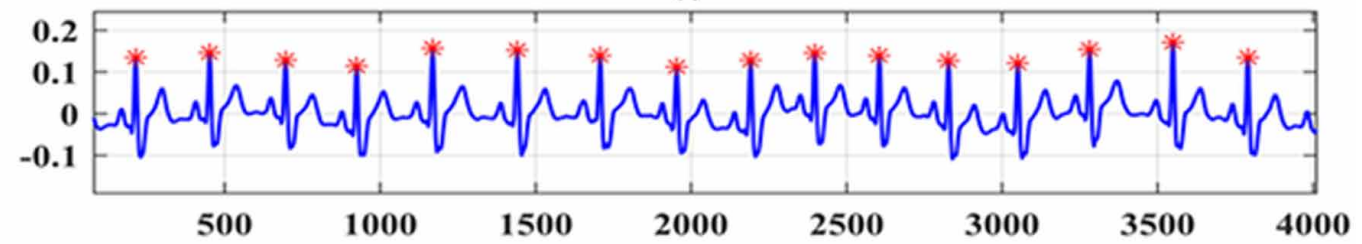

(e)

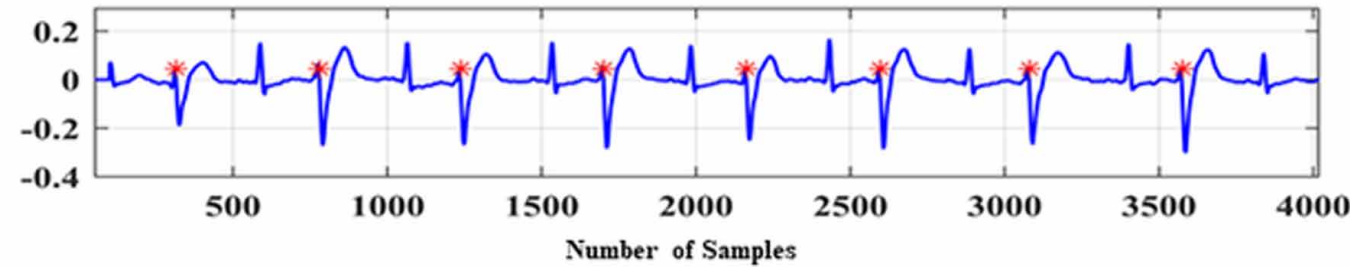

\subsubsection{Extreme Learning Machine (ELM)}

ELM is inspired by a biological structure, demonstrating that there is no need to tune hidden neurons in the network. Like a human brain, they are self-learner and trainers. Few merits of using ELM as supervised classifier are

- Direct solution for binary and multi-classification.

- Give solutions for both real and complex domains.

- Robust universal approximation and classification capability.

- Fast learner and classifier. 
Other from classification and regression, ELM finds its applications in areas like clustering, compression, and feature selection. ELM is also known as a feed-forward neural network with a single hidden layer.

ELM structure consists of input layers of $N$ dimensions and a hidden layer of $I$ nodes. Equation 9 displays ELM's output in initial stages as

$\sum_{x=1}^{N} h_{x}(u) \beta_{x}=v_{x}, x=1 \ldots \ldots . . N$

where $u_{x}=\left[u_{x 1}, u_{x 2} \ldots \ldots u_{x n}\right]^{T} \in \Re^{p}, v_{x}=\Re^{q}, x=1 \ldots \ldots . . N$ Or

$H=\beta T$

where $\beta$ is the output weights and $b=\left[b_{1}, b_{2}\right.$ $b_{I}{ }^{T}$, a vector of output weights. $T$ is the expected output and $H$ represents a matrix for the hidden layer as

$$
H=\left[\begin{array}{c}
h\left(u_{1}\right) \\
: \\
: \\
h\left(u_{n}\right)
\end{array}\right]=\left[\begin{array}{ccc}
h_{1}\left(x_{1}\right) & \ldots & h_{I}\left(u_{1}\right) \\
: & & : \\
: & & : \\
h_{1}\left(x_{n}\right) & \ldots & h_{I}\left(u_{n}\right)
\end{array}\right]
$$

$h(u)$ represents feature mapping of points from the $N$-dimensional domain to the $I$-dimensional domain (Su et al., 2017). The number of hidden nodes should be compared with the training sample points to increase ELM's memorizing capability.

\section{EXPERIMENTAL RESULTS}

As mentioned in the proposed work, Figure 5 displays R-peak detection in the filtered original heartbeats and Figure 6 shows the ECG extracted beats and their simulation. Also, Figure 7 displays six prime LDA coefficients of 2000 beats. The simulation results are obtained using MATLAB (R2016 working environment) on a Core i7 platform using window 10, 64-bit operating system.

\subsection{Classifier Performance Analysis}

The classifiers' performances get evaluated using standard confusion matrix statistics - Overall Accuracy Analysis (OAA), Sensitivity Analysis (SeA), Positive Predictivity Analysis (PpA), and Specificity Analysis (SpA). Table 3 presents classifier outcomes using a holdout cross-validation scheme. In this scheme, the partition divides the entire dataset into training and testing (holdout) subset according to the mentioned scalar parameter. For the current study, the parameter used is 0.30 , i.e., out of 2000 beats 1400 beats for the training phase, and 600 beats for the testing phase.

$$
O A A(\%)=\frac{T P N+T N N}{T P N+T N N+F P N+F N N} \times 100 \%
$$


Figure 6. Segmented and Generated ECG Beats: a) Normal b) Paced c) LBBB d) RBBB e) PVC
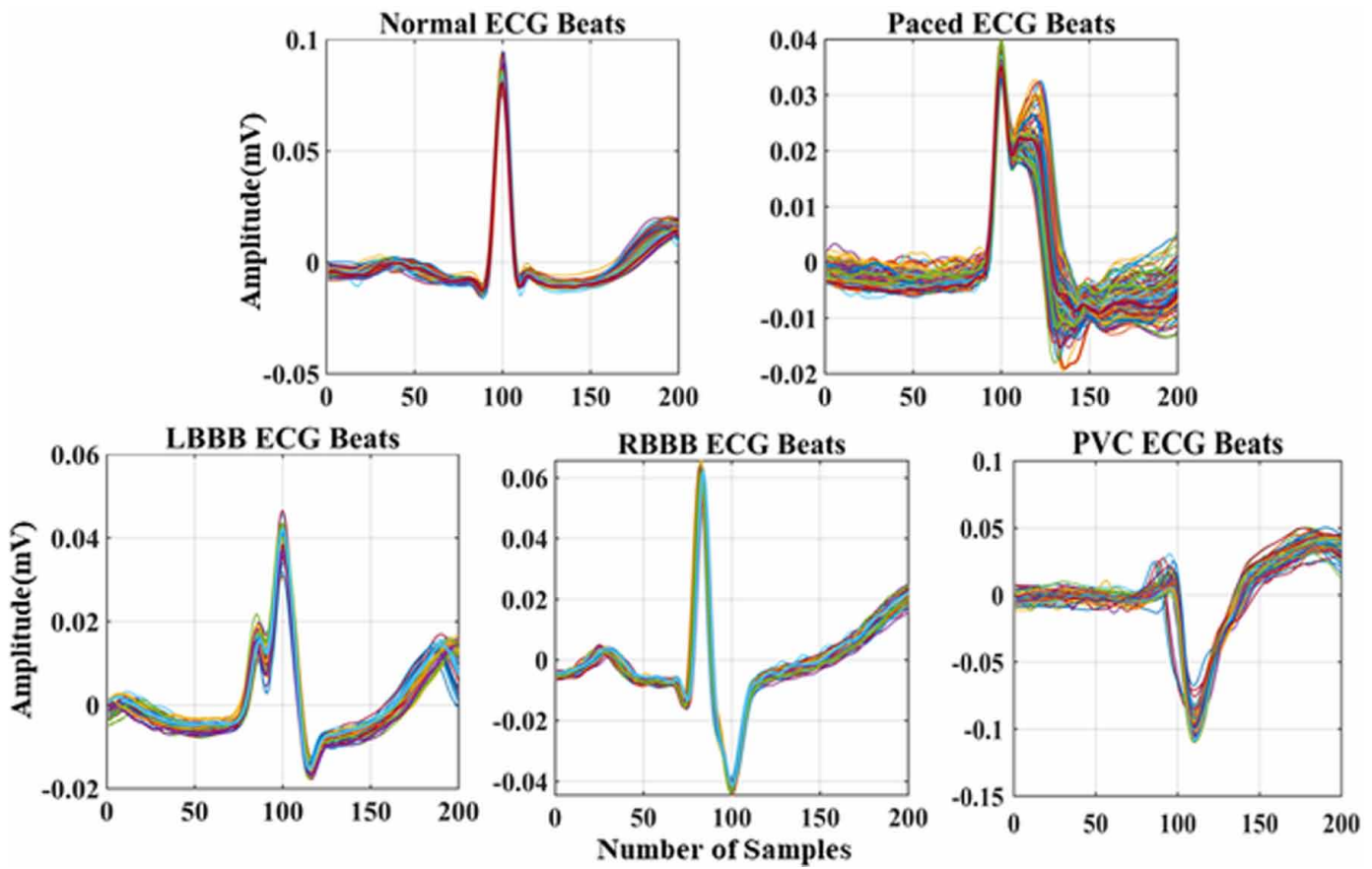

$\operatorname{SeA}(\%)=\frac{T P N}{T P N+F N N} \times 100 \%$

$P p A(\%)=\frac{T P N}{T P N+F P N} \times 100 \%$

$S p A(\%)=\frac{T N N}{T N N+F P N} \times 100 \%$

where TPN: True Positive Number, TNN: True Negative Number, FPN: False Positive Number and FNN: False Negative Number. The other vital parameter included in the classifier (training + testing) time, which specifies the proposed system's computational cost. It depends on the type and size of the dataset. The testing time helps in predicting the new unknown classification based on learning. In this current experiment, KNN and SVM have shown $99.8 \%$ of overall accuracy showing one misclassification, whereas ELM has shown $97 \%$ of it. The testing time of 600 beats in one instance shown by KNN, SVM, and ELM is $0.0234 \mathrm{~s}, 0.0081 \mathrm{~s}$, and $0.0036 \mathrm{~s}$. Figure 8 displays the SVM analysis of multi-class arrhythmia, and Figure 9 displays the performance representation of the classification.

The conclusion is that all three implemented classification modules have characteristics that can be further used for different cardiac applications. The first classifier KNN performance is well suited for mass ECG checking in hospitals in remote are. The next classifier i.e., ELM, is based on neuron 

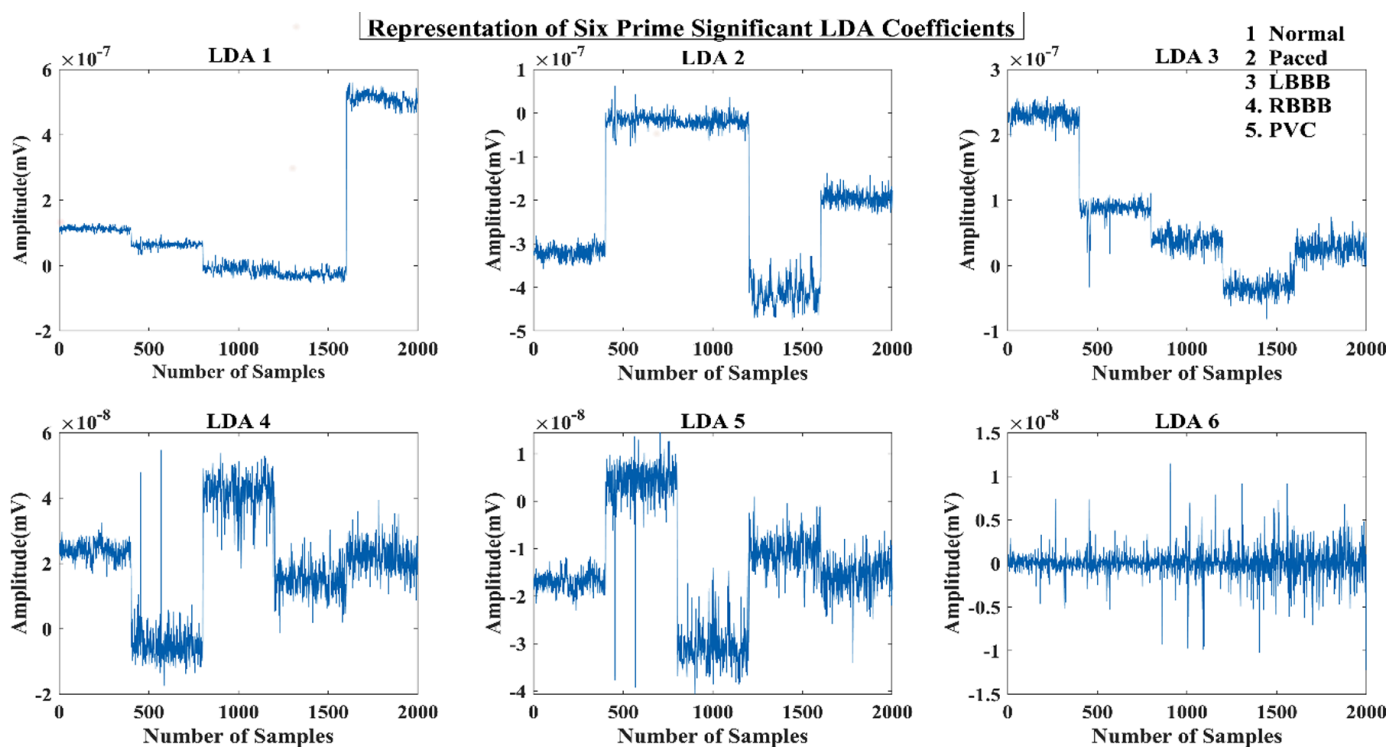

concept with a single hidden layer. With the help of bias and weights, the ELM output supports fast track and mobile smart e-health services. Furthermore, the latter approach is of SVM that helps in fast and accurate decisions e.g., emergencies. Figure 10 demonstrates the application part of the classifiers.

\subsection{Application-Specific Choice of Classification}

Nowadays, ECG analysis is required for maximum medical tests. Sometimes this requirement becomes very urgent, and sometimes it is just required to check if the ECG is normal or abnormal. Surveying the literature and taking opinion from medical experts, some suggested application modules have been listed as

1. Cardiac Health Camps: These camps are generally aided by charity or by social means. And are camped mostly in rural areas. ECGs to be checked up are more in numbers. According to the need, processor, computational cost and memory management have to be adjusted. The installation of hardware should also be in the budget. So, KNN, which shows high accuracy with less learning time, is best suited for this kind of application.

2. Mobile/Fast Track Devices: This service now comes under e-Health and is very important for personal use like in gyms while traveling, swimming, etc. The client can check his/her ECG on personal devices such as mobile phones and smartwatches. These devices have internet facilities to get connected to e-health services and doctors like silicon-based biomedical sensors (Xu, Y. et al., 2019). To provide fast access, ELM is best suited for checking ECG normality or abnormality at the client end. This will save the user time as well as the clinician time. High-speed processors are already involved in this scenario.

3. Intensive Care Unit (ICU) and Ambulance: This is a crucial application that has to be minutely taken care of. One medical expert is specially assigned to take care of this type of condition. So very accurate, stable, and fast classifier like SVM is required. Moreover, these areas are highly equipped with the latest technology. 


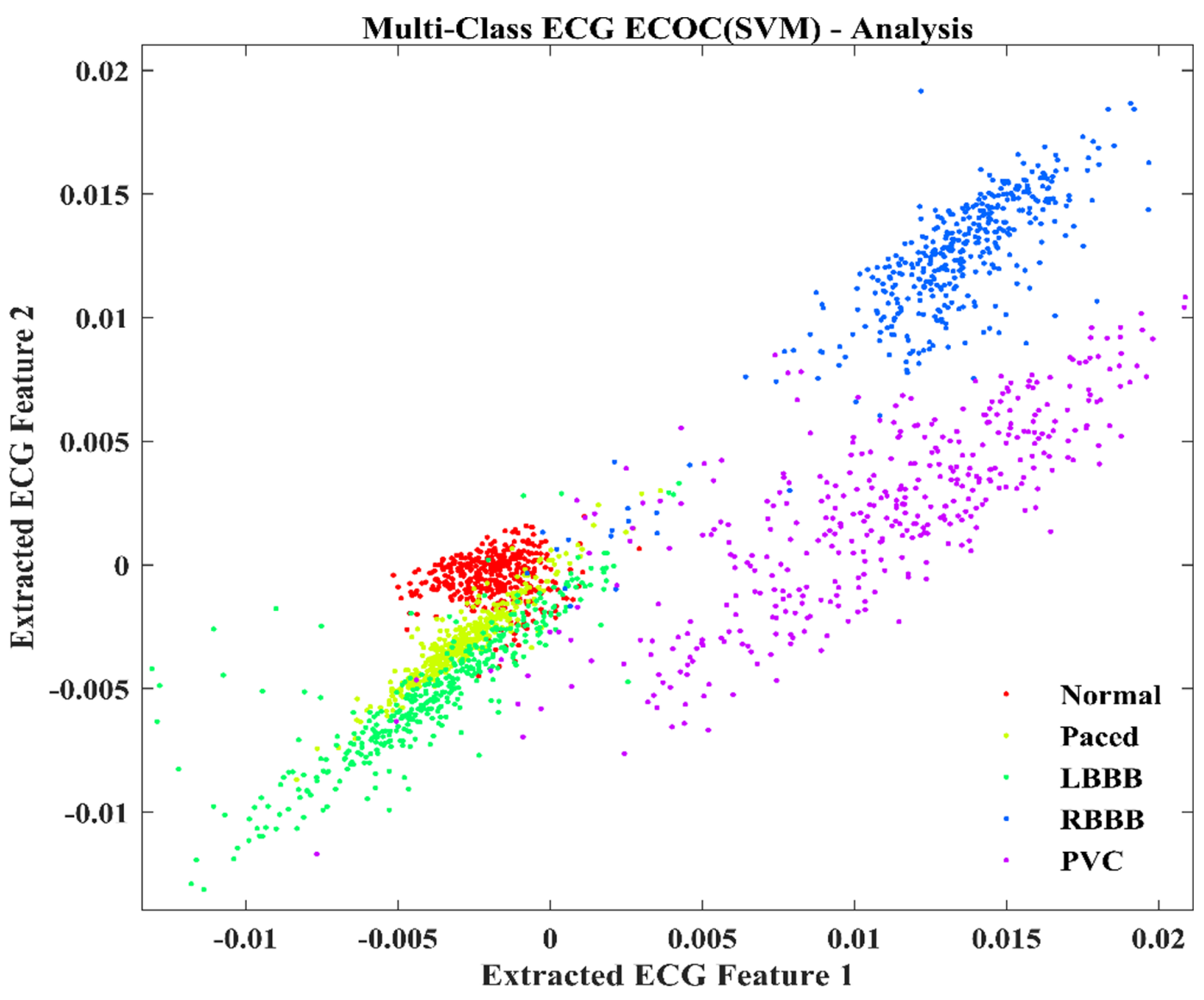

\section{DISCUSSION}

The performance review of proposed work with reported literature techniques working in a similar environment addresses the overall accuracy achieved. Table 4 tabulates the comparison with five existing studies. An automated ECG system has been developed using a discrete wavelet and PCA for the individual patient (Ince et al., 2009). They gained an accuracy of 95.58\% using Multidimensional Particle Swarm Optimization (MDPSO). The linear and non-linear ECG features are obtained from MIT/BIH database by applying DWT with PCA, HOS with ICA, and SVM-RBF as a classifier to obtain maximum accuracy 98.91\% (Elhaj et al., 2016). An ECG feature matrix with 1800 heartbeats is being utilized for DWT with KICA and PCA feature extraction and reduction (Li et al., 2016). The LIB-SVM is applied to a multi-domain ECG feature matrix to achieve an accuracy of $98.8 \%$. The multi-resolution characteristics in the wavelet domain have been explored for four beat ECG classification using neural network and SVM, achieving 96.69\% and 98.39\% of accuracy (Sahoo et al., 2017). Similarly, another existing literature is reported using the multi-resolution property of discrete wavelet and statistical features for non-fixed ECG signals (Yildirim, 2019). They obtained an accuracy of $98.51 \%$ using OSELM (Online Sequential - ELM).

The current study shows the achievement of the proposed combination of DTCWT and LDA implemented ECG feature dataset with powerful classification techniques of KNN, ELM, and SVM. The implementation of DTCWT double decomposition improves SNR and results in robust feature 
Figure 9. a) Classification Performance b) Classifier Testing Time

(a)

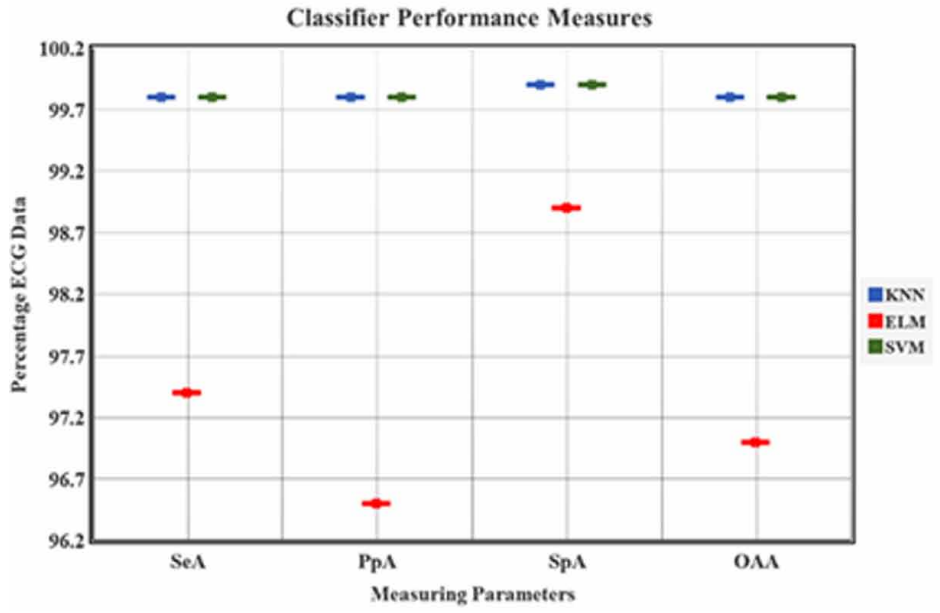

(b)

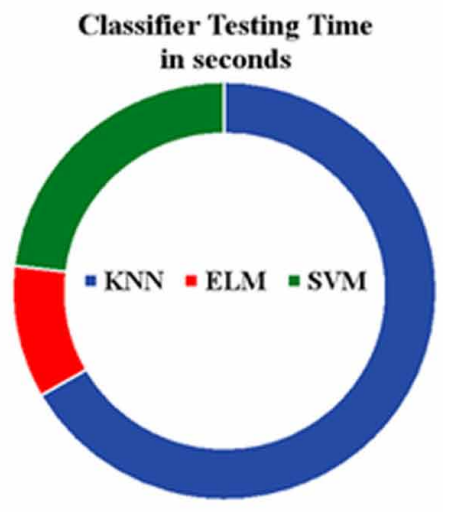

Table 3. Performance comparison of KNN, ELM and SVM based on ECG featured dataset

\begin{tabular}{|c|c|c|c|c|c|c|c|c|c|c|c|c|}
\hline \multirow{3}{*}{$\begin{array}{c}\text { Methods } \\
\text { KNN }\end{array}$} & \multirow{3}{*}{$\begin{array}{c}\begin{array}{c}\text { Types of } \\
\text { Beats }\end{array} \\
\text { Normal }\end{array}$} & \multicolumn{5}{|c|}{$\begin{array}{l}\text { Number of } \\
\text { Correct Classification }\end{array}$} & \multirow{2}{*}{\multicolumn{2}{|c|}{$\begin{array}{l}\text { Classifier (Time) } \\
\text { In Seconds } \\
\text { Training Testing }\end{array}$}} & \multirow{3}{*}{$\begin{array}{c}\mathrm{SeA} \\
\% \\
100\end{array}$} & \multirow{3}{*}{$\begin{array}{c}\text { PpA\% } \\
100\end{array}$} & \multirow{3}{*}{$\begin{array}{c}\text { SpA } \\
\% \\
100\end{array}$} & \multirow{3}{*}{$\begin{array}{c}\text { OAA, } \\
\% \\
99.8\end{array}$} \\
\hline & & \multirow{3}{*}{$\begin{array}{c}\text { TPN } \\
132 \\
111\end{array}$} & \multirow{2}{*}{$\frac{\text { FPN }}{0}$} & \multirow{2}{*}{$\frac{\text { FNN }}{0}$} & \multirow{2}{*}{$\frac{\text { TNN }}{468}$} & \multirow{3}{*}{$\begin{array}{c}\text { Total } \\
599 \\
\text { Average }\end{array}$} & & & & & & \\
\hline & & & & & & & & \multirow[t]{5}{*}{0.0234} & & & & \\
\hline & Paced & & 1 & 0 & 488 & & & & 100 & 99.1 & 99.8 & \\
\hline & LBBB & 111 & 0 & 1 & 488 & & & & 99.1 & 100 & 100 & \\
\hline & RBBB & 122 & 0 & 0 & 478 & & & & 100 & 100 & 100 & \\
\hline & PVC & 123 & 0 & 0 & 473 & & & & $\begin{array}{r}100 \\
99.8\end{array}$ & $\begin{array}{r}100 \\
99.8\end{array}$ & $\begin{array}{r}100 \\
99.9\end{array}$ & \\
\hline \multirow[t]{5}{*}{ ELM } & Normal & 131 & 1 & 1 & 467 & \multirow{5}{*}{$\begin{array}{c}582 \\
\text { Average }\end{array}$} & \multirow[t]{5}{*}{0.0428} & \multirow[t]{5}{*}{0.0036} & 99.2 & 99.2 & 99.8 & \multirow[t]{5}{*}{97} \\
\hline & Paced & 108 & 4 & 0 & 488 & & & & 100 & 96.4 & 99.2 & \\
\hline & LBBB & 107 & 4 & 7 & 482 & & & & 93.8 & 96.4 & 98.0 & \\
\hline & RBBB & 118 & 4 & 2 & 476 & & & & 98.3 & 96.7 & 99.2 & \\
\hline & PVC & 118 & 8 & 5 & 469 & & & & $\begin{array}{l}95.9 \\
97.4\end{array}$ & $\begin{array}{l}93.6 \\
96.5\end{array}$ & $\begin{array}{l}98.3 \\
98.9\end{array}$ & \\
\hline \multirow[t]{5}{*}{ SVM } & Normal & 132 & 0 & 0 & 468 & \multirow{5}{*}{$\begin{array}{c}599 \\
\text { Average }\end{array}$} & \multirow[t]{5}{*}{0.0994} & \multirow[t]{5}{*}{0.0081} & 100 & 100 & 100 & \multirow[t]{5}{*}{99.8} \\
\hline & Paced & 111 & 1 & 0 & 488 & & & & 100 & 99.1 & 99.8 & \\
\hline & LBBB & 111 & 0 & 1 & 488 & & & & 99.1 & 100 & 100 & \\
\hline & RBBB & 122 & 0 & 0 & 478 & & & & 100 & 100 & 100 & \\
\hline & PVC & 123 & 0 & 0 & 473 & & & & $\begin{array}{r}100 \\
99.8\end{array}$ & $\begin{array}{r}100 \\
99.8\end{array}$ & $\begin{array}{r}100 \\
99.9\end{array}$ & \\
\hline
\end{tabular}

extraction. The classification testing time has encouraged the whole module to end up for diverse applications in e-health services for frequent users and medical experts. 
Table 4. An abridgment of arrhythmia classification showing performance comparison

\begin{tabular}{|c|c|c|c|}
\hline \multirow[t]{2}{*}{ ECG Literature } & \multicolumn{2}{|c|}{ Arrhythmia Classification Structure } & \multirow[t]{2}{*}{ OAA } \\
\hline & Feature Extraction & Classifier & \\
\hline Ince et al., 2009 & DWT with PCA & MDPSO & 95.58 \\
\hline Elhaj et al., 2016 & $\begin{array}{l}\text { DWT with PCA } \\
+ \text { HOS with ICA }\end{array}$ & $\begin{array}{l}\text { SVM- } \\
\text { RBF }\end{array}$ & 98.91 \\
\hline Li et al., 2016 & $\begin{array}{c}\text { DWT with KICA } \\
\text { and PCA. }\end{array}$ & SVM & 98.8 \\
\hline Sahoo et al., 2017 & DWT & SVM & 98.39 \\
\hline Yildirim, 2019 & $\begin{array}{c}\text { DWT with } \\
\text { statistical features }\end{array}$ & OSELM & 98.51 \\
\hline Proposed work & DTCWT with LDA & $\begin{array}{l}\text { KNN } \\
\text { ELM } \\
\text { SVM }\end{array}$ & $\begin{array}{c}99.8 \\
97 \\
99.8\end{array}$ \\
\hline
\end{tabular}

Figure 10. Classification modules showing respective cardiac applications

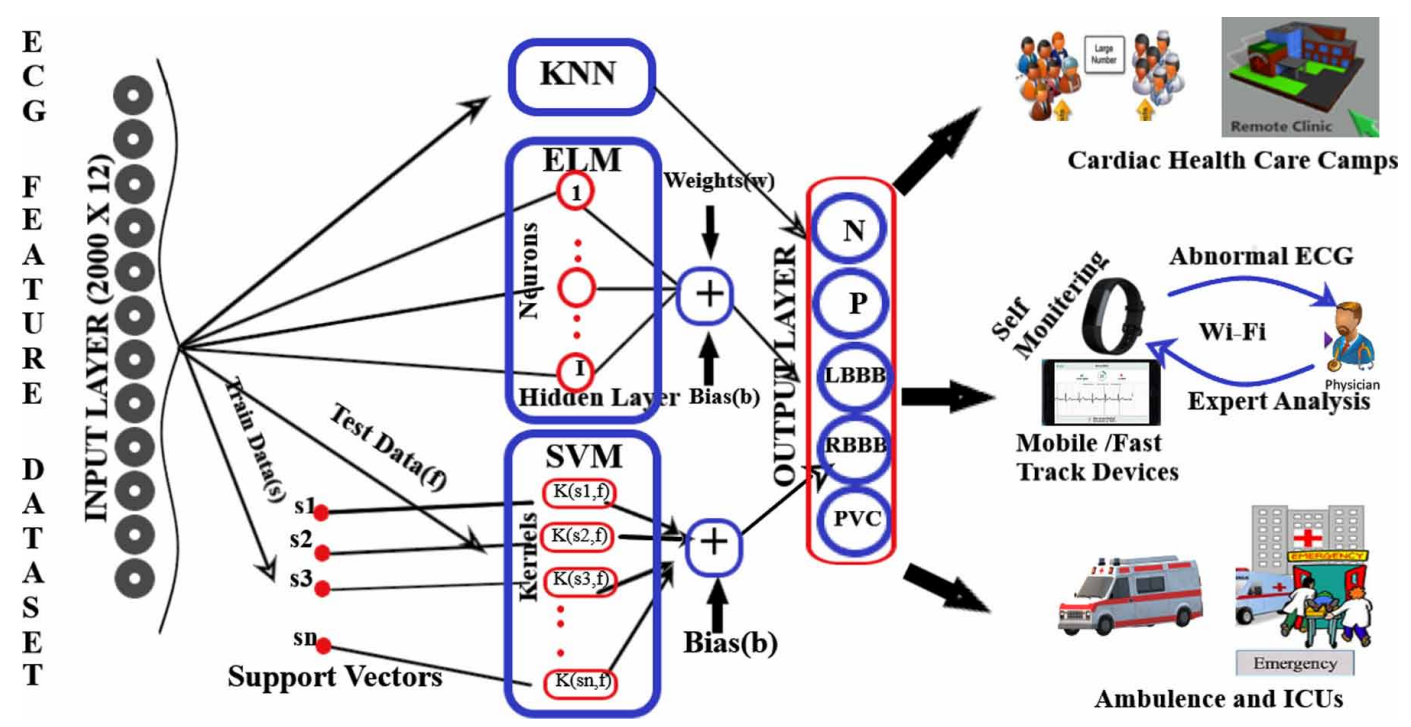

\section{CONCLUSION AND FUTURE SCOPE}

This paper aims at lossless feature extraction, compatible feature reduction, efficient and effective classification to develop application-specific arrhythmia detection modules instead of a generalized system for different medical requirements. The paper uses a hybrid approach consisting of DTCWT double decomposition and LDA to have a compact input ECG feature dataset for classifiers like KNN, 
ELM, and SVM. The performance evaluation is done on the MIT/BIH database. With shift-invariant, directional selectivity applied on ECG data and discrimination of inter and intra class distances, the classification operations of KNN and SVM both have shown $99.8 \%$ accuracy with $0.0234 \mathrm{~s}$ and $0.0081 \mathrm{~s}$ of testing time respectively. And for the same extracted ECG dataset, ELM has shown 97\% accuracy with $0.0036 \mathrm{~s}$ of testing time. This makes ELM results faster than SVM and KNN. Getting the desired accuracy and computational time all three classification modules are suggested for medical applications in consultancy with medical experts.

Further, in future more enhanced work can be shown for compression of ECG data and performance outcomes for mobile e- health care system as well as for secrecy and security of human ECG. 


\section{REFERENCES}

Acharya, U. R., Fujita, H., Oh, S. L., Hagiwara, Y., Tan, J. H., \& Adam, M. (2017). Application of deep convolutional neural network for automated detection of myocardial infarction using ECG signals. Information Sciences, 415, 190-198. https://doi.org/10.1016/j.ins.2017.06.027

Acharya, U. R., Sudarshan, V. K., Koh, J. E. W., Martis, R. J., Tan, J. H., Oh, S. L., Muhammad, A., Hagiwara, Y., Mookiah, M. R. K., \& Chua, K. P. et al. (2017). Application of higher-order spectra for the characterization of coronary artery disease using electrocardiogram signals. Biomedical Signal Processing and Control, 31, 31-43. https://doi.org/10.1016/j.bspc.2016.07.003

Afonso, V. X., Tompkins, W. J., Nguyen, T. Q., \& Luo, S. (1999). ECG beat detection using filter banks. IEEE Transactions on Biomedical Engineering, 46(2), 192-202. https://doi.org/ 10.1109/10.740882

Duin, R. P. W., \& Loog, M. (2004). Linear dimensionality reduction via a heteroscedastic extension of LDA: The Chernoff criterion. IEEE Transactions on Pattern Analysis and Machine Intelligence, 26(6), 732-739. https:// doi.org/10.1109/TPAMI.2004.13

El B'charri, O., Latif, R., Elmansouri, K., Abenaou, A., \& Jenkal, W. (2017). ECG signal performance de-noising assessment based on threshold tuning of dual-tree wavelet transform. Biomedical Engineering Online, 16(1), 26. https://doi.org/10.1186/s12938-017-0315-1

Elhaj, F. A., Salim, N., Harris, A. R., Swee, T. T., \& Ahmed, T. (2016). Arrhythmia recognition and classification using combined linear and nonlinear features of ECG signals. Computer Methods and Programs in Biomedicine, 127, 52-63. https://doi.org/10.1016/j.cmpb.2015.12.024

Georgieva-Tsaneva, G., \& Tcheshmedjiev, K. (2013). Denoising of electrocardiogram data with methods of wavelet transform. In International Conference on Computer Systems and Technologies (Vol. 13, pp. 9-16). Academic Press.

Gunn, S. R. et al. (1998). Support vector machines for classification and regression. ISIS Technical Report, 14(1), 5-16.

Inan, O. T., Giovangrandi, L., \& Kovacs, G. T. A. (2006). Robust neural-network-based classification of premature ventricular contractions using wavelet transform and timing interval features. IEEE Transactions on Biomedical Engineering, 53(12), 2507-2515. https://doi.org/10.1109/TBME.2006.880879

Ince, T., Kiranyaz, S., \& Gabbouj, M. (2009). A generic and robust system for automated patient-specific classification of ECG signals. IEEE Transactions on Biomedical Engineering, 56(5), 1415-1426. https://doi. org/10.1109/TBME.2009.2013934

Kaur, I., Rajni, R., \& Marwaha, A. (2016). ECG signal analysis and arrhythmia detection using wavelet transform. Journal of The Institution of Engineers (India): Series B, 97(4), 499-507. 10.1007/s40031-016-0247-3

Kingsbury, N. (2001). Complex wavelets for shift invariant analysis and filtering of signals. Applied and Computational Harmonic Analysis, 10(3), 234-253.

Li, H., Yuan, D., Wang, Y., Cui, D., \& Cao, L. (2016). Arrhythmia classification based on multi-domain feature extraction for an ECG recognition system. Sensors (Basel), 16(10), 1744. https://doi.org/10.3390/s16101744

Martis, R. J., Acharya, U. R., \& Min, L. C. (2013). ECG beat classification using PCA, LDA, ICA and discrete wavelet transform. Biomedical Signal Processing and Control, 8(5), 437-448. https://doi.org/10.1016/j. bspc.2013.01.005

Minami, K., Nakajima, H., \& Toyoshima, T. (1999). Real-time discrimination of ventricular tachyarrhythmia with Fourier-transform neural network. IEEE Transactions on Biomedical Engineering, 46(2), 179-185. https:// doi.org/10.1109/10.740880

Mirvis, D. M., \& Goldberger, A. L. (2001). Electrocardiography. Heart Disease, 1, 82-128.

Mitra, S., Mitra, M., \& Chaudhuri, B. B. (2006). A rough-set-based inference engine for ECG classification. IEEE Transactions on Instrumentation and Measurement, 55(6), 2198-2206. https://doi.org/10.1109/TIM.2006.884279

Moody, G. B., \& Mark, R. G. (2001). The impact of the MIT-BIH arrhythmia database. IEEE Engineering in Medicine and Biology Magazine, 20(3), 45-50. https://doi.org/10.1109/51.932724 
Oh, S. L., Ng, E. Y. K., San Tan, R., \& Acharya, U. R. (2018). Automated diagnosis of arrhythmia using combination of CNN and LSTM techniques with variable length heart beats. Computers in Biology and Medicine, 102, 278-287. https://doi.org/10.1016/j.compbiomed.2018.06.002

Pławiak, P. (2018). Novel methodology of cardiac health recognition based on ECG signals and evolutionaryneural system. Expert Systems with Applications, 92, 334-349. https://doi.org/10.1016/j.eswa.2017.09.022

Prashar, N., Sood, M., \& Jain, S. (2018). Semiautomatic detection of cardiac diseases employing dual tree complex wavelet transform. Periodicals of Engineering and Natural Sciences, 6(2), 129-140. 10.21533/pen.v6i2.188

Raj, S., Maurya, K., \& Ray, K. C. (2015). A knowledge-based real time embedded platform for arrhythmia beat classification. Biomedical Engineering Letters, 5(4), 271-280. https://doi.org/ 10.1007/s13534-015-0196-9

Sahoo, S., Kanungo, B., Behera, S., \& Sabut, S. (2017). Multiresolution wavelet transform based feature extraction and ECG classification to detect cardiac abnormalities. Measurement, 108, 55-66. https://doi.org/10.1016/j. measurement.2017.05.022

Shadmand, S., \& Mashoufi, B. (2016). A new personalized ECG signal classification algorithm using blockbased neural network and particle swarm optimization. Biomedical Signal Processing and Control, 25, 12-23. https://doi.org/10.1016/j.bspc.2015.10.008

Singh, R., Mehta, R., \& Rajpal, N. (2018). Efficient wavelet families for ECG classification using neural classifiers. Procedia Computer Science, 132, 11-21. j.procs.2018.05.05410.1016/

Singh, R., Rajpal, N., \& Mehta, R. (2019). Abnormality detection in ECG using hybrid feature extraction approach. In 2018 First International Conference on Secure Cyber Computing and Communication. ICSCCC. https://doi.org/10.1109/ICSCCC.2018.8703349.

Singh, R., Rajpal, N., \& Mehta, R. (2020). Wavelet and kernel dimensional reduction on arrhythmia classification of ECG signals. EAI Endorsed Transactions on Scalable Information Systems: Online First. 10.4108/eai.13-72018.163095

Su, H., Cai, Y., \& Du, Q. (2017). Firefly-algorithm-inspired framework with band selection and extreme learning machine for hyperspectral image classification. IEEE Journal of Selected Topics in Applied Earth Observations and Remote Sensing, 10(1), 309-320. https://doi.org/10.1109/JSTARS.2016.2591004

Thomas, M., Das, M. K., \& Ari, S. (2015). Automatic ECG arrhythmia classification using dual tree complex wavelet based features. AEÜ. International Journal of Electronics and Communications, 69(4), 715-721. https:// doi.org/10.1016/j.aeue.2014.12.013

Venkatesan, C., Karthigaikumar, P., \& Varatharajan, R. (2018). A novel LMS algorithm for ECG signal preprocessing and KNN classifier based abnormality detection. Multimedia Tools and Applications, 1-10. $10.1007 / \mathrm{s} 11042-018-5762-6$

Xu, Y., Hu, X., Kundu, S., Nag, A., Afsarimanesh, N., Sapra, S., Mukhopadhyay, S. C., \& Han, T. (2019). Siliconbased sensors for biomedical applications: A review. Sensors (Basel), 19(13), 2908. https://doi.org/10.3390/ s19132908

Yildirim, Ö. (2019). Ecg beat detection and classification system using wavelet transform and online sequential elm. Journal of Mechanics in Medicine and Biology, 19(1), 1940008. 10.1142/S0219519419400086 
Ritu Singh is a research scholar in Guru Gobind Singh Indraprastha University(GGSIPU). She received her B.E. (Electronics) from Poona University, Maharashtra, and M.E (Electronics and Communication) from Maharishi Dayanand Univerity (MDU), Rohtak. She has more than 8 years of teaching experience. Her current research interests include signal processing, soft computing, ECG, and machine learning algorithms She has several publications in reputed international journals and conferences.

Navin Rajpal is a Professor at USICT since September 2004. He served as Dean, USICT from October 1, 2011, to September 30, 2014. He completed his BSc (Engineering) in Electronics and Communication from the R.E.C. Kurukshetra, now known as an NIT, Kurukshetra. He completed his MTech and PhD from Computer Science and Engineering Department, IIT, Delhi. He served in various capacities and has more than 31 years of experience in teaching and research. He has supervised several MTech and 12 Ph.D. students. He has published/presented more than 100 research papers in national and international journals/conferences. He is a life member of CSI and ISTE. His areas of interest are computer vision, image processing, pattern recognition, artificial neural networks, computer graphics, algorithms design, and digital hardware design.

Rajesh Mehta is working as an Assistant Professor in the Thapar Institute of Engineering and Technology in the Computer Science and Engineering Department. He received his Ph.D. in Information Technology from Guru Gobind Singh Indraprastha University (GGSIP) and M. Tech. in Computer Science \& Engineering (CSE) from Guru Jambheshwar University, Hisar. He has teaching and research experience of more than 14 Years. He has published and presented more than 22 papers in SCl-indexed journals and International conferences. His current research interests include image processing, signal processing, digital watermarking, machine learning algorithms, genetic algorithm, and fuzzy logic. 